\title{
A new component of solar dynamics: North-South diverging flows migrating toward the equator with an 11 year period
}

\author{
J. G. Beck \\ JBeck@solar.Stanford.EDU \\ W. W. Hansen Experimental Physics Laboratory, \\ Stanford University, Stanford, CA 94305-4085 \\ L. Gizon \\ LGizon@solar.Stanford.EDU \\ W. W. Hansen Experimental Physics Laboratory, \\ Stanford University, Stanford, CA 94305-4085 \\ and \\ T. L. Duvall, Jr. \\ Laboratory for Astronomy and Solar Physics, \\ NASA, Goddard Space Flight Center \\ Greenbelt, MD 20771, USA \\ TDuvall@solar.Stanford.EDU
}

\begin{abstract}
Time-distance helioseismology analysis of dopplergrams provides maps of torsional oscillations and meridional flows. Meridional flow maps show a timevarying component that has a banded structure which matches the torsional oscillations with an equatorward migration over the solar cycle. The time-varying component of meridional flow consists of a flow diverging from the dominant latitude of magnetic activity. These maps are compared with other torsional oscillation maps and with magnetic flux maps, showing a strong correlation with active latitudes. These results demonstrate a strong link between the time-varying component of the meridional flow and the torsional oscillations.
\end{abstract}

Subject headings: Sun: atmospheric motions - Sun: rotation 


\section{Introduction}

Torsional oscillations and meridional flows have been studied for over two decades (eg. Duvall 1979, LaBonte and Howard 1982, Ulrich et al 1988). The meridional flow is generally agreed to have a poleward flow in both hemispheres with an amplitude of $10-20 \mathrm{~m} \mathrm{~s}^{-1}$; the torsional oscillations (also termed 'zonal flows') consist of latitudinal bands of alternating faster and slower rotation which migrate toward the equator over the solar cycle and are superimposed on top of the differential rotation. The time-distance helioseismology studies of meridional and torsional oscillations conducted by Giles et al (1997), and Giles (1999) showed that meridional flows extend through the convection zone, and torsional oscillations extend to at least $35 \mathrm{Mm}$ below the surface.

Howard \& LaBonte (1980) showed torsional oscillations as a 5-10 $\mathrm{m} \mathrm{s}^{-1}$ flow pattern superimposed on differential rotation which migrates toward the equator over the solar cycle similar to the "butterfly diagram" showing latitudes of magnetic activity. Recently, helioseismic techniques have been applied to probe the depth extent of torsional oscillations (eg. Kosovichev \& Schou, 1997; Schou et al. 1998). The flows have been seen to extend to depths of $56 \mathrm{Mm}$ (Howe et al 2000). Further, Beck \& Schou (2000) have seen torsional oscillation patterns in supergranulation motions which suggest that these flows extend to the depth of supergranulation.

Meridional flows have been difficult to measure by direct Doppler observation due to their small amplitude and the large amplitude velocity patterns such as convective limb shift and rotation. However, Nibes, Meunier and Vince (1997) have studied meridional flows using sunspots as tracers and have identified a co-variance of East-West and North-South motions consistent with angular momentum transport which would sustain differential rotation. Time variations of the meridional flow were noticed by Ulrich et al, (1998), and Hathaway, (1996). Haber et al (2002) have studied meridional flows using ring-diagram analysis. They have found indications of a second meridional cell during the years 1998 through 2001 in the outer $7 \mathrm{Mm}$. Additionally, they found an asymmetry in the meridional flows between the northern and southern hemispheres which would have impact on angular momentum transport. Chou \& Dai (2001) have studied subsurface meridional flows using time-distance helioseismology and found a time-varying component which extends down to $70 \mathrm{Mm}$. It was suggested that the varying flow could be linked to magnetic activity. 


\section{Description of Data and Reduction Techniques}

The data reduction followed the description in Giles (1999). The data spanned from May, 1996 through July, 2001 and consist of medium-resolution Dopplergrams obtained from the MDI instrument (Scherrer et al, 1995) on board the spacecraft SOHO. The gap in data from June 1998 until March 1999 corresponds to the period of broken contact with SOHO. Images were grouped into 72-hour periods for de-trending of solar rotation and supergranulation. Regions, spanning $100^{\circ}$ degrees in latitude and longitude, were tracked with solar rotation for the 72 -hour period. These tracked regions were 'stacked' into a data cube for further processing, which consisted of applying a high-pass filter (cutoff of $1.7 \mathrm{mHz}$ ) to remove the residual supergranule signal and performing a phase-velocity filter to select modes with a range in $\omega / k_{h}$; where $\omega$ was the temporal frequency and $k_{h}$ was the horizontal wave-number.

Once the data had been prepared, temporal cross-correlations were obtained using code developed by Giles (1999). The code calculated correlations between pairs of points displaced in a roughly North-South direction to detect meridional flows, the deviation from the North-South direction varied by up to $30^{\circ}$. This procedure was performed in a roughly East-West direction to detect torsional oscillations. When using a pair of points that are not exactly North-South or East-West, a second pair was used that cancels the effect of orthogonal flows. The pairs of points were separated by an amount varying from $3^{\circ}$ to $45^{\circ}$ in latitude or longitude. To improve the signal-to-noise, the resulting cross-correlation functions were averaged over all observed longitudes and saved to files for each nominal latitude. Subsequently, the cross-correlation function for each latitude was averaged over three-month periods. Further averaging was done over the ranges of latitudes, keeping the mid-point constant, producing 61 latitude bins, with a width of $\sim 3$ degrees. This was repeated for 21 three month epochs.

\section{Analysis and Results}

The time delay of the wave was found by fitting to the cross-correlation function. A positive time delay corresponds to a flow to the North in the case of meridional flows or a flow to the West for East-West flows.

The measurement of the travel time shifts due to flows follows closely the method discussed by Gizon and Birch (2002). At each distance, a mean reference cross correlation symmetric for the two senses of time lag was derived. This reference function was cross correlated with an individual cross correlation to yield an approximately zero-centered result 
except for the shift away from zero due to the flow. A weighted average over distance was derived with the contribution peaked near a distance of $17^{\circ}$ with a width of $4^{\circ}$. A ray connecting points separated by $17^{\circ}$ extends to a depth of $65 \mathrm{Mm}$. The time shift was measured as twice the shift of the maximum of this correlation away from zero lag by forcing a parabola through the three closest points to the peak. The factor of two is necessary to match earlier definitions of time shift which involved measuring the shift of positive time lags and negative time lags separately and taking the difference, effectively doubling the signal.

The meridional flow is strongly anti-symmetric (shown in Figure 1), with positive values indicating flows in the northward direction. To study the anti-symmetric component separately, the flow measured at southern latitudes was subtracted from the measurements at northern latitudes.

The East-West flow was similarly analyzed, with the exception that the symmetric component was subtracted from the signal to produce the torsional oscillation pattern shown in Figure 2b. Torsional oscillations obtained from $f$-modes by Schou et al (2002) are shown for comparison.

A map of magnetic field strength is included as Figure $2 \mathrm{~d}$ to indicate the active latitudes over the period of interest. To compare with the flow maps, a mean latitude of magnetic activity was derived corresponding to the calendar quarters of the velocity analysis by averaging the absolute value of MDI magnetic synoptic charts for the appropriate Carrington rotations, re-binning in latitude and longitude to again match the velocity analysis, applying a three-point $(0.25,0.5,0.25)$ smoothing in both latitude and longitude, symmetrizing in latitude, squaring and fitting a Gaussian function in latitude at each longitude. The location of the peak of the Gaussian is then the mean latitude of activity. The fit was done to the square of the field strength so that the background function would be inconsequential. The curve of mean latitude of activity is plotted on top of the velocity images in Figure 2 to indicate the strong connection between the activity and the equatorward propagating flow pattern.

The flows are organized about the mean latitude of activity. The North-South flow is away from this latitude, implying an up-flow to conserve mass. Whether this up-flow causes the magnetic activity to emerge at this latitude or is a result of rising magnetic flux is unknown. The torsional oscillation is faster equatorward of the mean activity latitude and slower poleward, as noted previously by LaBonte \& Howard (1982). From the p-mode studies, we know that the torsional oscillation persists at depths of at least $56 \mathrm{Mm}$. In the present work, we have not derived the depth dependence of the north-south component explicitly, but we might expect it to persist over a significant fraction of the depth over which the $p$ modes used extend. 


\section{Discussion}

The time-varying component of the meridional flow correlates very well with the torsional oscillations, with a more strongly poleward flow corresponding to a slower rotation. The lanes between bands of stronger or weaker flow match for both the meridional and torsional oscillations and these lanes align with the mean latitude of magnetic activity.

This correlation between these flows and magnetic activity may be interpreted as a result of rising material, accompanied by rising flux tubes, diverging at the photosphere. Whether the diverging North-South flow is a cause or an effect of the rising flux tubes is unknown.

However, it should be noted that the co-variation of the zonal and time-varying meridional flows is not consistent with the Coriolis effect acting upon rising material. Rather, the covariance is consistent with angular momentum transport toward the equator.

Another interpretation could be that these time-varying flows are long-term averages of outflows from active regions.

\section{Acknowledgments}

We thank J. Schou who kindly provided data for comparison, as well as R. K. Ulrich

for useful discussions. This work was supported by NASA grant NAG5-8878. SOHO is a project of international cooperation between ESA and NASA.

\section{REFERENCES}

Beck, J.G., \& Schou, J., 2000, Sol. Phys., 193, 333

Chou, D., \& Dai, D., 2001, ApJ, 559, L175

Duvall, T. L., Jr. 1979, Sol. Phys., 63, 3

Giles, P.M., Duvall, T.L. Jr., Scherrer, P.H., Bogart, R.S., 1997, Nature, 390, 52

Giles, P. M., 1999, PhD thesis, Stanford Univ

Gizon, L. \& Birch, A. C., 2002, ApJ, in press

Haber, D.A., et al, 2002., submitted

Hathaway, D., ApJ, 460, 1027.

Howard, R. \& LaBonte, B. 1980, ApJ, 239, L33 
Howe, R., et al, ApJ, 533, L163

Kosovichev, A.G. \& Schou, J., 1997, ApJ, 482, L207

LaBonte B. \& Howard, 1982, Sol. Phys., 63, 373

Nesme-Ribes, E., Meunier, N., Vince, I., 1997, å, 321, 323

Scherrer, P.H., et al, 1995, Sol. Phys., 162, 129

Schou, J, et al, 1998, ApJ, 505, 390

Schou, J, 1999, ApJ, 523, L181

Ulrich, R.K. 1988, Sol. Phys., 117, 291

\section{Captions}

Fig 1 - Mean Meridonal Flow. The mean signal of meridional flows is shown here for the interval May 1996 - July 2001. A northward flow has a positive sign. From a simple meridional flow model (Giles, 1999), a scale was obtained that $10 \mathrm{~m} / \mathrm{s}$ corresponds to 0.5 second time delay for this distance.

Fig 2- Meridional flow and torsional oscillation maps from time-distance and a torsional oscillation map from $f$ modes, compared with a butterfly diagram of magnetic fields. The heavy black line over-plotted is the mean latitude of activity. a) The poleward flow, obtained from subtracting a fit to the mean meridional flow found using time-distance techniques and symmetrizing the residuals. A positive time delay indicates a poleward flow in both hemispheres. b)The torsional oscillations obtained from time-distance techniques. c) Torsional oscillations obtained from $f$ modes (Schou, 1999). d) Mean magnetic field derived from MDI synoptic charts. 


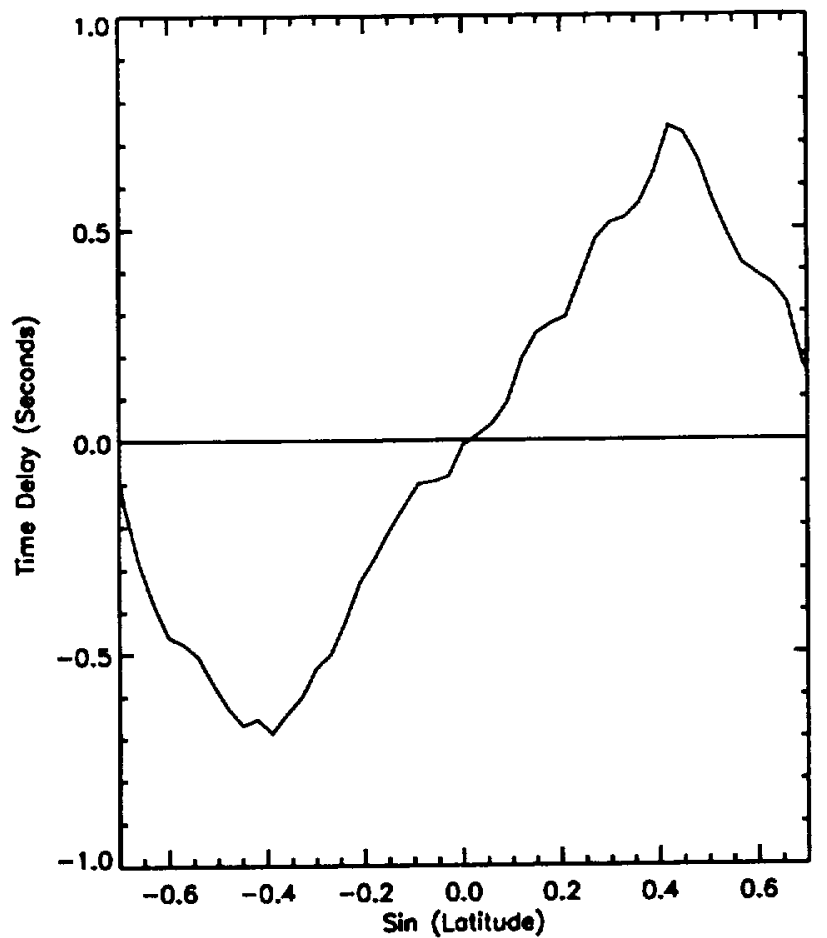

Fig. 1.- Mean Meridional Flow. 

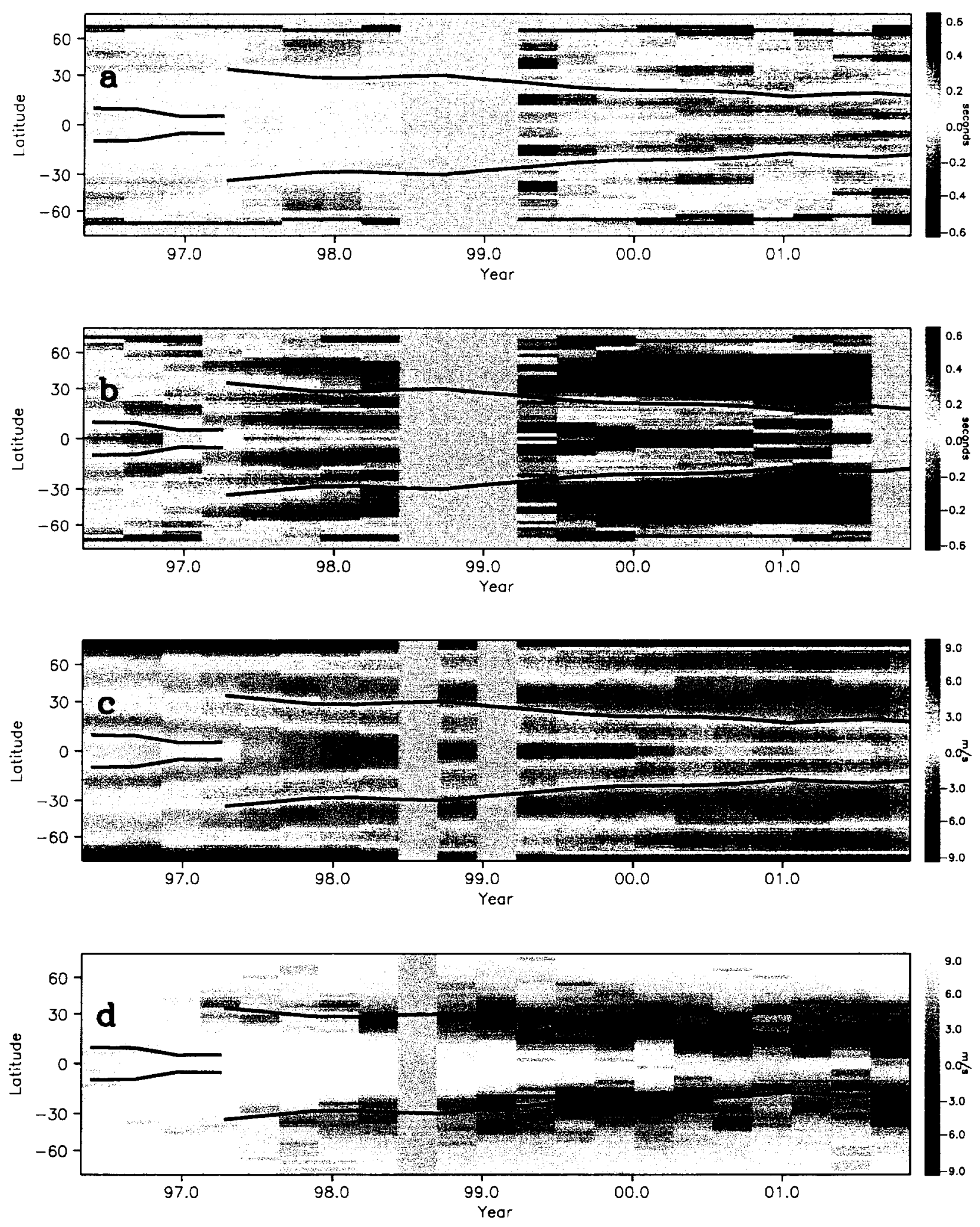

Fig. 2.- Meridional flow and torsional oscillation maps 